\title{
Efficacy of Electrical Stimulation-Augmented Virtual Reality Training in Improving Balance in Individuals with Incomplete Spinal Cord Injury: Study Protocol of a Randomized Controlled Trial
}

\author{
Shefali Walia ${ }^{1,2}$, Pragya Kumar $^{1}$, Chitra Kataria ${ }^{2}$ \\ ${ }^{1}$ Amity Institute of Physiotherapy, Amity University Uttar Pradesh, Noida, India \\ ${ }^{2}$ ISIC Institute of Rehabilitation Sciences, Indian Spinal Injuries Centre, New Delhi, India
}

Study Design: This study is a single-blind, parallel, three-group, and randomized controlled trial.

Purpose: This study aimed to investigate the effectiveness of electrical stimulation-augmented virtual reality training in improving balance in individuals with incomplete spinal cord injury (iSCI).

Overview of Literature: Individuals with iSCl often face significant balance and mobility impairments affecting their quality of life. Scientific studies focusing on standing balance training in the iSCI population are limited. Virtual reality-based balance training has shown positive results in several neurological populations. Electrical stimulation has also proved to be effective in improving voluntary muscle strength in partially paralyzed muscles after iSCl as well as promoting neuroplasticity.

Methods: Forty-eight iSCI participants will be recruited based on the inclusion criteria. The participants will be randomly assigned to any of the three groups: virtual reality-based balance training along with the electrical stimulation group, virtual reality-based balance training along with sham stimulation group, or virtual reality-based balance training group. The intervention will be delivered as 60-minute sessions, thrice a week for 4 weeks.

Results: The performance of the participants will be assessed using the lower extremity motor score, static and dynamic balance assessment using TechnoBody ProKin tilting platform and Berg Balance Scale, Walking Index for Spinal Cord Injury, and World Health Organization Quality of Life-BREF at pre-intervention, after 4 weeks post-intervention, and at 1-month follow-up.

Conclusions: The trial will provide new knowledge about the effectiveness of electrical stimulation-augmented virtual reality training in improving balance in individuals with iSCl. The study results will contribute to the design of better rehabilitation programs for individuals with iSCl.

Keywords: Spinal cord injury; Balance rehabilitation; Virtual reality; Electrical stimulation

\section{Introduction}

Spinal cord injury (SCI) is one of the most catastrophic injuries of the nervous system resulting in permanent neurological deficits, functional impairments, and abrupt change in the quality of the person's life. It may lead to a

Received Feb 5, 2020; Revised Sep 8, 2020; Accepted Sep 30, 2020

Corresponding author: Pragya Kumar

Amity Institute of Physiotherapy, Amity University, Sector 125, Noida, Uttar Pradesh, 201303, India

Tel: +91-78529787, E-mail: pkumar24@amity.edu 
profound disability with negative physiological, physical, or psychological sequelae and restricted employment opportunities even after the individual has reintegrated into community living [1]. The global incidence of SCI is estimated to range between eight and 246 cases per million persons [2]. Epidemiological studies have shown that approximately $50 \%$ of individuals with traumatic spinal cord damage suffer from an incomplete lesion [3].

The recovery of walking function is one of the primary aims of rehabilitative programs in individuals with incomplete SCI (iSCI) [4]. However, impairments in muscle strength, sensation, and abnormal muscle tone often make it difficult to regain walking function [5]. Balance is one of the most important factors affecting walking in chronic SCI individuals and is often ignored during rehabilitation [4].

Research specifying effective balance training protocols for individuals with iSCI is limited. Prior studies have examined the effects of visual feedback [6], divided attention stepping tasks [7], backward walking [8], body weightsupported treadmill training [9], biofeedback [10], and virtual reality (VR) training [11]. However, no randomized controlled trials have been conducted until currently to identify the most effective standing balance training protocol for the iSCI population.

Rehabilitation methods based on VR have been recently introduced and have shown positive results for balance training in several neurological populations [12]. VR provides the advantage of creating multiple sensory inputs such as visual, sensory, and auditory feedback and taskspecific approaches by creating a virtual environment that is similar to that of the real world. VR provides a usercomputer interface that implements real-time simulation of activity or environment and allows user interaction via multiple sensory modalities, hence, augmenting subjects' attention and motivation [13]. Previous studies based on game-based exercises and VR have shown to improve dynamic sitting balance in the SCI population $[14,15]$.

Electrical stimulation (ES) has often been used as a therapeutic tool to promote recovery of function following nervous system injury [16]. Moreover, ES has been used to improve voluntary muscle strength in the partially paralyzed muscles in SCI [17]. Animal studies with chronic SCI have proved that an electrically stimulated exercise of the lower limbs enhances new neural cell birth and survival [16]. This demonstrates that ES can enhance cellular regeneration in the injured adult central nervous system and raises the possibility of using controlled elec- trical activation of the nervous system for optimizing spontaneous regeneration and functional recovery in neurologic injuries [18].

The main objective of this study is to investigate the efficacy of ES-augmented VR training protocol for improving lower extremity muscle strength, balance, functional mobility, and quality of life (QOL) in persons with iSCI. A follow-up study 1-month post-intervention shall also be conducted. It is hypothesized that significant improvement in lower extremity muscle strength, balance, functional mobility, and QOL of persons with iSCI will be noticeable after 4 weeks of ES-augmented VR training when compared with participants receiving VR training alone. In addition, these improvements shall be maintained after 1-month post-intervention as well.

\section{Materials and Methods}

A clinical trial is designed to see the efficacy of the protocol on lower extremity muscle strength, balance, functional mobility, and QOL in persons with iSCI.

\section{Patient and study design}

A single-blind, parallel, three-group, and randomized controlled trial will be undertaken. A purposive sample of 48 participants with iSCI will be recruited from the inpatient and outpatient rehabilitation departments of the Indian Spinal Injuries Centre, New Delhi, India. All participants will be provided with information sheets, and written informed consent will be obtained by an investigator before recruitment. The demographic details will be obtained, and the participants will be selected based on eligibility criteria after the neurological examination (Table 1). Medical clearance from the treating doctor is taken for all participants before enrolment into the intervention. The criteria for discontinuation included participant requests or reporting of adverse events to the investigator.

The participants will be randomly assigned to any of the three groups: virtual reality-based balance training+electrical stimulation (VR-ES) group, virtual reality-based balance training+sham stimulation (VRSS) group, or virtual reality-based balance training (VR) group for thrice a week for 4 weeks. The recruitment will be over 2 years. This research protocol followed the Standard Protocol Items: Recommendations for Interventional Trials (SPIRIT) recommendations (SPIRIT schedule) (Ta- 
Table 1. Eligibility criteria

\begin{tabular}{|c|c|}
\hline & Criteria \\
\hline \multirow[t]{6}{*}{ Inclusion criteria } & $\begin{array}{l}\text { Traumatic or non-traumatic spinal cord injury categorized as American Spinal Injury Association Impairment Scale grade C or D since at least } 6 \\
\text { months }\end{array}$ \\
\hline & Level of injury from C5 to L1 \\
\hline & Age between $18-45$ years \\
\hline & Able to stand with or without the support of an assistive device for a minimum of 2 minutes \\
\hline & Willing to participate in the study \\
\hline & Mini-Mental Status Examination scores $\geq 24$ \\
\hline \multirow[t]{9}{*}{ Exclusion criteria } & Spinal shock \\
\hline & Any other pre-existing neurological problem, musculoskeletal condition, or psychiatric problem which may interfere with the treatment \\
\hline & Pain scored greater than 5 on an 11-point numerical rating pain scale (0, no pain; 10 , most severe pain) \\
\hline & Any other pre-existing recent complication that can interfere with participation \\
\hline & Symptoms of orthostatic hypotension upon standing \\
\hline & Severe depression (Beck Depression Inventory-II $\geq 29$ ) \\
\hline & Spasticity of grade 3 or more on Modified Ashworth Scale \\
\hline & Visual or auditory impairment such that it impacts on the ability to participate \\
\hline & $\begin{array}{l}\text { Additional exclusion criteria for ES group includes contraindications for ES such as: (1) cardiac pacemakers; (2) skin lesions or rash at potential } \\
\text { sites; (3) grade } 4 \text { pressure ulcers anywhere on the lower extremities according to the National Pressure Ulcer Advisory Panel classification; } \\
\text { and (4) grade } 2 \text { or } 3 \text { pressure ulcers according to the National Pressure Ulcer Advisory Panel classification at locations where ES is to be ap- } \\
\text { plied. }\end{array}$ \\
\hline
\end{tabular}

ES, electrical stimulation.

Table 2. Schedule of enrollment, interventions, and assessments according to the Standard Protocol Items: Recommendations for Interventional Trials guideline

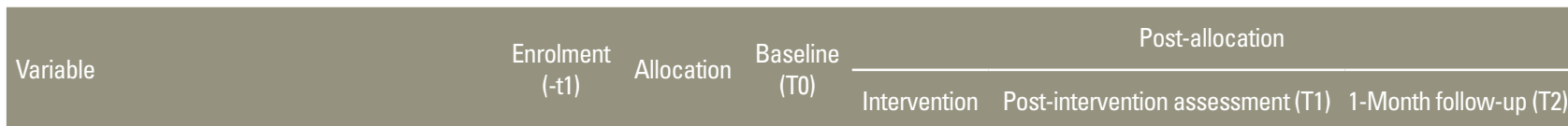

Enrolment

\begin{tabular}{|c|c|c|c|c|}
\hline Eligibility screen & $x$ & & & \\
\hline Informed consent & $x$ & & & \\
\hline Medical clearance & $x$ & & & \\
\hline Allocation & & & & \\
\hline \multicolumn{5}{|l|}{ Interventions } \\
\hline VR-ES group & & & & \\
\hline VR-SS group & & & & \\
\hline VR group & & & & \\
\hline \multicolumn{5}{|l|}{ Assessments } \\
\hline Lower extremity motor score & $x$ & $x$ & $x$ & $x$ \\
\hline Limits of stability & $x$ & $x$ & $x$ & $x$ \\
\hline Dynamic balance tests & $x$ & $x$ & $x$ & $x$ \\
\hline Berg Balance Scale & $x$ & $X$ & $x$ & $x$ \\
\hline Walking Index for Spinal Cord Injury & $x$ & $x$ & $x$ & $x$ \\
\hline World Health Organization Quality of Life-BREF & $X$ & $X$ & $x$ & $x$ \\
\hline
\end{tabular}

VR-ES group, virtual reality-based balance training along with electrical stimulation; VR-SS group, virtual reality-based balance training along with sham stimulation; VR group, virtual reality-based balance training only. 
ble 2). A visual description of the study regarding study enrolment, assessments, interventions, and follow-up is shown in Table 2. Baseline assessments (T0) are taken before allocation, which consists of blocking randomization (1:1:1 ratio). Post-intervention assessments of the three groups (T1) serve to compare the short-term efficacy of the different interventions. Follow-up assessments after 1 month (T2) would be done to detect potential long-term effects. The planned experimental design is consistent with current Consolidated Standards of Reporting Trials (CONSORT) guidelines (Fig. 1).

\section{Ethical considerations}

All potential trial participants will be informed orally and in writing about the purpose of this trial, its potential risks, costs and benefits of participation, and the right to withdraw before the screening.

A written informed consent would be taken and signed by the participants who are interested for participation in the study. The study protocol has been approved by the in- stitutional review board of the Indian Spinal Injuries Centre and Amity University and registered with the Clinical Trials Registry-India (CTRI/2018/12/016814). Data will be collected, managed, stored, and confidentiality maintained. All authors will have access to the final trial dataset. During the study, the participants shall not be allowed to participate in other rehabilitation or research interventions that can influence the outcome of the study.

\section{Randomization}

Randomization for enrolled participants will be performed by a computer-generated randomization sequence created using the Random Allocation Software by a person other than the principal investigator. The allocation schedule will be sequentially numbered and sealed in opaque envelopes to ensure concealment. Furthermore, a person not associated with the study will sequentially open the numbered envelopes to reveal the participant's group allocation upon completion of each participant's assessment. The participants will be assigned to one of the
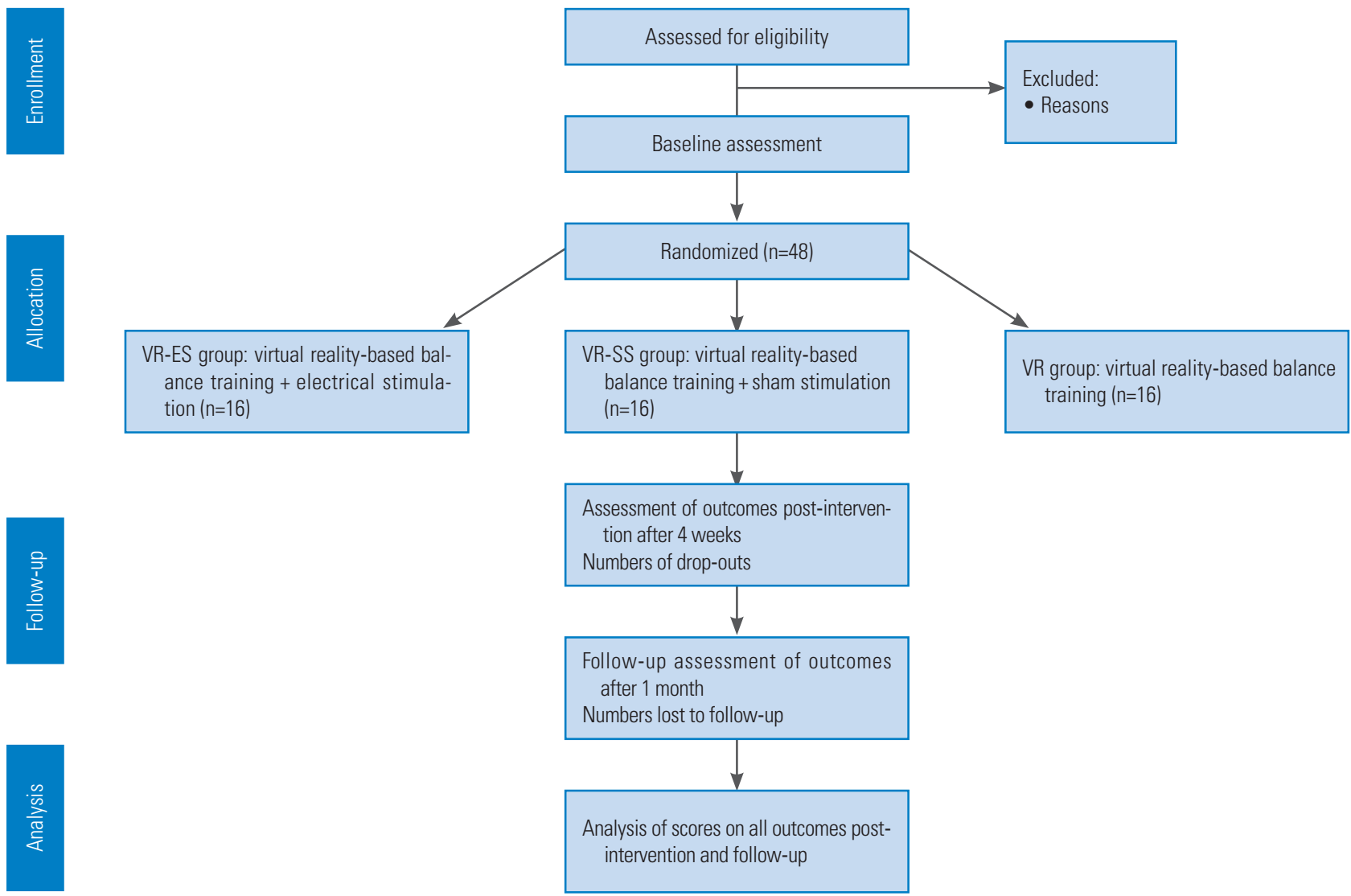

Fig. 1. CONSORT (Consolidated Standards of Reporting Trials) flowchart. VR-ES group, virtual reality-based balance training along with electrical stimulation; VR-SS group, virtual reality-based balance training along with sham stimulation; VR group, virtual reality-based balance training only. 
VR-ES, VR-SS, or VR groups.

\section{Blinding}

The outcome assessor will be blinded to group allocation. On the other hand, the principal investigator will be informed of the group allocation given the nature of the interventions. Investigators who will perform data analysis would be unaware of the treatment group.

\section{Intervention}

The intervention program will be divided into three phases (Table 3). VR training is provided by the Wii Fit Plus software and the Wii Fit balance board system (Nintendo Co. Ltd., Kyoto, Japan). It uses a central game console connected to a $127-\mathrm{cm}$ (50-inch) screen wall-mounted television. Navigation is via the handheld control pad and physical whole-body movements through standing on the Wii Fit balance board controlled by an avatar in the gaming environment. The choice of games was based on the similarity between the training and real-life conditions and based on previous research using the Nintendo Wii in training balance in various neurological populations (Table 4) $[12,19,20]$. During the first session, the therapist shall demonstrate the game controls and instructions to ensure that the participants understand how to control the system. Furthermore, the therapist shall offer verbal and manual guidance to all participants to help them learn the best strategies to reach the highest scores in each game. A walker shall be placed in front of the participant if required for safety. The duration of each game varies from 1 to 2 minutes. The exercises shall be presented in a similar order. An equal number of rounds for each exercise shall

Table 3. Details of intervention in each group

\begin{tabular}{|c|c|}
\hline Intervention phase & Details of intervention \\
\hline Phase 1 (15 min) & Kinesiotherapy: stretching, free exercises, light resistance exercises, and postural adjustments followed by joint mobility \\
\hline \multicolumn{2}{|l|}{ Phase 2 (30 min) } \\
\hline VR-ES group & $\begin{array}{l}\text { Virtual reality-based balance training by the Wii Fit Plus software and Wii Fit balance board system + electrical stimulation on bilateral quad- } \\
\text { riceps, hamstrings, dorsiflexors, and plantarflexors }\end{array}$ \\
\hline VR-SS group & Virtual reality-based balance training by the Wii Fit Plus software and Wii Fit balance board system + sham stimulation \\
\hline VR group & Virtual reality-based balance training by the Wii Fit Plus software and Wii Fit balance board system \\
\hline Phase 3 (15 min) & $\begin{array}{l}\text { Learning transfer activities: participants to perform real-world exercises that included movements based on those practiced while engaging in } \\
\text { virtual reality tasks, such as: weight shifting in the left and right directions and then progressing to forward and backward weight shifting } \\
\text { (resembling penguin slide) while standing in front of the mirror placed } 2 \mathrm{~m} \text { away, straight line walking (resembling tightrope), and heading a } \\
\text { ball thrown at them by the therapist in various directions (resembling soccer heading). }\end{array}$ \\
\hline
\end{tabular}

VR-ES group, virtual reality-based balance training along with electrical stimulation; VR-SS group, virtual reality-based balance training along with sham stimulation; VR group, virtual reality-based balance training only.

Table 4. Description of games using Wii Fit

\begin{tabular}{|c|c|c|}
\hline Game & General description & Therapeutic goals \\
\hline Tightrope walking & $\begin{array}{l}\text { Standing on a Wii balance board, subject shifts weight from side to side to } \\
\text { mimic walking in place. Player must jump over obstacles by slightly extend- } \\
\text { ing knees while maintaining balance. }\end{array}$ & $\begin{array}{l}\text { Weight shift to left and right for dynamic balance attention } \\
\text { and coordination; motor response; visual and auditory } \\
\text { feedback }\end{array}$ \\
\hline Soccer heading & $\begin{array}{l}\text { Standing on a Wii balance board, subjects move their weight in the direction } \\
\text { in which a ball is flying. The avatar heads the ball. Attention is paid to } \\
\text { avoid hitting objects that are not balls. }\end{array}$ & $\begin{array}{l}\text { Weight shift to left and right for dynamic balance attention } \\
\text { and coordination; motor response; visual and auditory } \\
\text { feedback }\end{array}$ \\
\hline Penguin slide & $\begin{array}{l}\text { Standing on a Wii balance board, subjects with slight knee flexion shift their } \\
\text { body left and right to catch as many fish as possible without falling off the } \\
\text { iceberg. }\end{array}$ & $\begin{array}{l}\text { Weight shift to left and right for dynamic balance postural } \\
\text { control; attention and coordination; visual and auditory } \\
\text { feedback }\end{array}$ \\
\hline Balance bubble & $\begin{array}{l}\text { Standing on the Wii balance board, subject navigates down the river in a } \\
\text { bubble by weight shifting in all planes. The participant has to avoid being } \\
\text { hit by walls, rocks, and stinging bee. }\end{array}$ & $\begin{array}{l}\text { Weight shift to left and right+forward and backward for dy- } \\
\text { namic balance postural control; attention and coordination; } \\
\text { visual and auditory feedback }\end{array}$ \\
\hline Table tilt & $\begin{array}{l}\text { Standing on the Wii balance board, subjects guide balls into holes by shift- } \\
\text { ing left, right, forward, and backward. Platform tilts along with the players } \\
\text { movement. }\end{array}$ & $\begin{array}{l}\text { Weight shift in multiple directions for dynamic balance pos- } \\
\text { tural control; attention and coordination; visual and audi- } \\
\text { tory feedback }\end{array}$ \\
\hline
\end{tabular}


be presented to the participants during each training session. Rest would be allowed and training would be resumed afterward if the subject declares fatigue.

Participants in the VR-ES group shall also receive ES while performing virtual balance exercises. ES shall be delivered using an eight-channel electrical stimulator that uses surface self-adhesive stimulation electrodes placed on the subject's skin corresponding to the muscles targeted. The key muscles that shall be targeted are the bilateral quadriceps, hamstrings, dorsiflexors, and plantarflexors. The pulse width is $50 \mu$ s and the pulse frequency is $20 \mathrm{~Hz}$. The current levels shall be separately selected for each muscle group such that the participant felt the stimulation without having the stimulated muscles contract due to the stimulation [21].

Participants randomized to the VR-SS group will be managed identically with the VR-ES group, but the amplitude shall be set at $0 \mathrm{~mA}$ so no ES shall occur while performing balance exercises on the Nintendo Wii. The participants shall be told before treatment that they may or may not feel the stimulation to promote a similar mental setup. Those in the VR group shall not receive any form of ES while performing virtual balance exercises.

\section{Progression and feedback}

The initial difficulty of the exercises shall be adjusted for each participant based on their performance during the familiarization session. The difficulty level of a game will be gradually increased to require more multidirectional movement in the center of the mass. The levels will be progressed when the participant reaches at least a 95\% success rate in three rounds. The participants will receive a concomitant visual and auditory knowledge of the results of the success of the task when movements are accurately made within a specified time because learning is also affected by performance-related feedback. On the other hand, participants will also receive negative knowledge of the performance of the use of compensatory strategies while performing games using the Wii Fit.

\section{Adherence}

The adherence of the participants to the training protocol will be monitored through documentation of session attendance, length, and activities. Participants in all groups shall receive VR-based balance training exercises which may positively influence adherence and reduce attrition rates because all groups are likely to feel engaged in an active intervention. The participants would be permitted to increase the training duration by 1 week keeping in mind the "intention-to-treat" principle if the participants will be unable to complete the total number of sessions within the stipulated 4 weeks. The participants will be monitored through regular telephonic reminders to ensure compliance in the follow-up. For missing data during followup, the principle of the "last observation carried forward" shall be applied, wherein the missing final values of the outcome variable are replaced by the last known value before the participant is lost to follow-up [22]. The authors also fully report the type, extent, and pattern of missing data throughout the study.

\section{Outcome variables}

\section{1) Lower extremity motor score}

The lower extremity motor score (LEMS), a subscale of the American Spinal Injury Association classification, is the sum of the bilateral lower extremity key muscle power, ranging from total paralysis (0) to normal active movement with a full range of motion against gravity and full resistance (5) with a total possible score of 50 [8]. The five key muscles (hip flexors, knee extensors, ankle dorsiflexors, long toe extensors, and ankle plantar flexors) of each leg will be assessed.

\section{2) Balance assessment}

ProKin tilting platform (ProKin PK254; TechnoBody Inc., Dalmine, Italy) will be used for the measurement of postural stability indexes. The TechnoBody is a monoaxial platform that consists of three strain gauges that are placed under a circular surface of $55 \mathrm{~cm}$ in diameter and have a $20-\mathrm{Hz}$ sampling frequency. Participants will be tested with two levels of postural difficulty including double-leg standing with opened and closed eyes. Participants will be instructed to stand still, barefoot with arms at side and feet $10 \mathrm{~cm}$ apart, look at a screen in front of them, and not to talk during the test [23]. The parameter for the assessment of static balance will be the limits of stability, and the dynamic balance in terms of the stability index. The anterior-posterior stability index is the stability index calculated around the vertical reference axis while the medial-lateral stability index is calculated around the horizontal reference axis. The total stability index is the 
vector sum between both anterior-posterior and mediallateral stability indices. A higher stability index represents more variability and decreased postural stability. A good reliability of Technobody Prokin has been established in physically active persons (intraclass correlation [ICC] range, 0.55-0.79) [24].

\section{3) Berg Balance Scale}

The Berg Balance Scale (BBS) is a clinical measure of balance consisting of 14 tasks of progressing difficulty, each graded on a five-point ordinal scale and then summed up to obtain a total score ranging from 0 to 56 . Scoring is based on the ability to meet a certain time or distance requirement and to perform the items without assistance. The participants shall wear their usual shoes and braces, if necessary, during testing. Assessments will be conducted without the participants' walking assistive devices [25]. The interobserver reliability for BBS has been reported to be excellent both for single items $(0.84-0.98 ; p<0.001)$ and for the total score (ICC, 0.95 ; $95 \%$ confidence interval, $0.910-0.975)$ [26].

\section{4) Walking Index for Spinal Cord Injury II}

The Walking Index for Spinal Cord Injury (WISCI) II is a walking assessment comprising of 21 levels representing a gradation of walking performance based on the need for physical assistance, braces, and walking aids. WISCI II ranges from 0 to 20 . The maximal level at which a person will be able to walk safely shall be recorded. Moreover, it has been reported to have excellent reproducibility (ICC, $0.995)$ and inter- and intra-rater reliability (1.00) in the SCI population [27].

\section{5) World Health Organization Quality of Life-BREF}

The World Health Organization Quality of Life-BREF (WHOQOL-BREF) is a 26-item instrument consisting of four domains (physical health, psychological health, social, and environmental) and two overall QOL and general health items. Respondents rated the intensity, frequency, or evaluation of the selected attributes of QOL during the previous 2 weeks on a 5 -point Likert-response scale. The mean score in each domain will be obtained by computing the mean of transformed scores converted to a 0-100 scale for each domain. A mean score of $<40,41-60$, and $>60$ in each domain denoted poor, moderate, and good QOL, respectively. All domains of the WHOQOLBREF have been reported to show good internal consis- tency (Cronbach a range, 0.74-0.78) except for the social relationships domain $(\alpha=0.54)$ in individuals with SCI [28]. The Hindi version of the same shall be used for participants who may have difficulty in filling out the English form of the WHOQOL-BREF [29].

\section{Statistics}

\section{1) Sample size}

Sample size estimation was done to test the improvements in the outcome measures in each of the three groups. An a priori sample size estimation was performed using the G*Power 3 software (v3.1.2-3.1.9; Heinrich-Heine-Universität Düsseldorf, Düsseldorf, Germany; http://www. gpower.hhu.de/). The total sample size was calculated at 42 by using an a level of 0.05 , a power of $80 \%$, and an allocation ratio of 1:1:1. A minimum of $10 \%$ improvement in scores was expected in the BBS [10]. The potential loss to attrition was estimated at $10 \%$ to $15 \%$ based on researches. The total sample size was 48 .

\section{2) Statistical methods}

The Student $t$-test for numerical variables and Pearson's chi-square test for categorical variables will be applied to verify whether the randomization process generated between each group of participants have homogenous clinical and demographic characteristics before the intervention, thus avoiding possible selection bias. Secondary analyses will be carried out with this variable as a covariate, and analysis will be done using an analysis of covariance model to take into account group differences at baseline for any of the variables such as level of the lesion.

Descriptive data will be exposed as means or proportions along with standard deviations. Continuous variables will be summarized as mean \pm standard deviation for normal distribution and median \pm interquartile range for non-normal distribution. The Shapiro-Wilk test will be used to ensure the normal distribution of data. For the variables with a normal distribution, outcomes between groups across periods will be compared using the $3 \times 3$ mixed-model analysis of variance. The intragroup comparison will be analyzed by paired Student $t$-test or Wilcoxon test (if data is not normally distributed). All statistical analysis will be performed following the intentionto-treat principle. All statistical tests will be implemented using IBM SPSS ver. 21.0 (IBM Corp., Armonk, NY, USA) with a bilateral inspection. The level of statistical signifi- 
cance is assumed at $p$-value $<0.05$.

\section{Results}

The performance of the participants will be assessed on LEMS, and the static and dynamic balance assessment using the TechnoBody ProKin tilting platform and BBS, WISCI, and World Health Organization QOL-BREF at pre-intervention, after 4 weeks post-intervention, and 1-month follow-up.

\section{Discussion}

The recovery of balance during standing is one of the primary and essential aims of rehabilitative programs in individuals with iSCI. Retraining standing balance is challenging for healthcare professionals dealing with SCI. The trial will provide information about the effectiveness of ESaugmented VR training in improving balance in persons with iSCI. It is believed that the results of this training will help to improve lower extremity muscle strength, balance, functional mobility, and QOL in persons with iSCI. Previous studies have identified the limitation of transfer of the gains acquired in the virtual balance task during the training session to the performance of tests of balance and stability outside the context of the trained task conditions [30]. The current study will include a third phase of the intervention that will comprise of learning transfer activities to practice the virtual balance task in the real world as well.

This trial is designed to meet the methodological demand for adequate randomization, allocation concealment, and blinding of outcome assessors and statisticians. The trial will be reported according to the CONSORT guidelines. A limitation of this trial is that it will be a single-blinded trial (assessor only) due to the nature of the intervention. The recruitment of the participants shall be done through a convenience sample from one rehabilitation center in New Delhi. This may result in a possible selection bias.

\section{Conclusions}

This protocol will help determine the efficacy of ESaugmented VR training protocol for improving standing balance and functional mobility in persons with iSCI. This is one of the few protocols which have been designed specifically for iSCI. Thus, it is believed that this protocol will result in substantial improvement in standing balance in individuals with incomplete SCI despite the limitations.

\section{Conflict of Interest}

No potential conflict of interest relevant to this article was reported.

\section{Author Contributions}

SW, PK, and CK conceived the idea for the study. All authors contributed to the design and development of the study. SW drafted the manuscript. All authors have critically reviewed and approved the final manuscript.

\section{References}

1. Mat Rosly M, Halaki M, Mat Rosly H, et al. Exergaming for individuals with spinal cord injury: a pilot study. Games Health J 2017;6:279-89.

2. Van den Berg ME, Castellote JM, Mahillo-Fernandez I, de Pedro-Cuesta J. Incidence of spinal cord injury worldwide: a systematic review. Neuroepidemiology 2010;34:184-92.

3. Jackson AB, Dijkers M, Devivo MJ, Poczatek RB. A demographic profile of new traumatic spinal cord injuries: change and stability over 30 years. Arch Phys Med Rehabil 2004;85:1740-8.

4. Scivoletto G, Romanelli A, Mariotti A, et al. Clinical factors that affect walking level and performance in chronic spinal cord lesion patients. Spine (Phila Pa 1976) 2008;33:259-64.

5. McDonald JW, Sadowsky C. Spinal-cord injury. Lancet 2002;359:417-25.

6. Sayenko DG, Alekhina MI, Masani K, et al. Positive effect of balance training with visual feedback on standing balance abilities in people with incomplete spinal cord injury. Spinal Cord 2010;48:886-93.

7. Leach SJ, Magill RA, Maring JR. Using a dividedattention stepping accuracy task to improve balance and functional outcomes in an individual with incomplete spinal cord injury: a case report. Physiother Theory Pract 2017;33:72-81.

8. Foster H, DeMark L, Spigel PM, Rose DK, Fox EJ. The effects of backward walking training on balance and mobility in an individual with chronic incomplete spinal cord injury: a case report. Physiother 
Theory Pract 2016;32:536-45.

9. Alexeeva N, Sames C, Jacobs PL, et al. Comparison of training methods to improve walking in persons with chronic spinal cord injury: a randomized clinical trial. J Spinal Cord Med 2011;34:362-79.

10. Tamburella F, Scivoletto G, Molinari M. Balance training improves static stability and gait in chronic incomplete spinal cord injury subjects: a pilot study. Eur J Phys Rehabil Med 2013;49:353-64.

11. Wall T, Feinn R, Chui K, Cheng MS. The effects of the Nintendo(TM) Wii Fit on gait, balance, and quality of life in individuals with incomplete spinal cord injury. J Spinal Cord Med 2015;38:777-83.

12. Robinson J, Dixon J, Macsween A, van Schaik P, Martin D. The effects of exergaming on balance, gait, technology acceptance and flow experience in people with multiple sclerosis: a randomized controlled trial. BMC Sports Sci Med Rehabil 2015;7:8.

13. Song YB, Chun MH, Kim W, Lee SJ, Yi JH, Park $\mathrm{DH}$. The effect of virtual reality and tetra-ataxiometric posturography programs on stroke patients with impaired standing balance. Ann Rehabil Med 2014;38:160-6.

14. Khurana M, Walia S, Noohu MM. Study on the effectiveness of virtual reality game-based training on balance and functional performance in individuals with paraplegia. Top Spinal Cord Inj Rehabil 2017;23:263-70.

15. Betker AL, Desai A, Nett C, Kapadia N, Szturm T. Game-based exercises for dynamic short-sitting balance rehabilitation of people with chronic spinal cord and traumatic brain injuries. Phys Ther 2007;87:1389-98.

16. Becker D, Gary DS, Rosenzweig ES, Grill WM, McDonald JW. Functional electrical stimulation helps replenish progenitor cells in the injured spinal cord of adult rats. Exp Neurol 2010;222:211-8.

17. De Freitas GR, Szpoganicz C, Ilha J. Does neuromuscular electrical stimulation therapy increase voluntary muscle strength after spinal cord injury?: a systematic review. Top Spinal Cord Inj Rehabil 2018;24:6-17.

18. Creasey GH, Ho CH, Triolo RJ, et al. Clinical applications of electrical stimulation after spinal cord injury. J Spinal Cord Med 2004;27:365-75.

19. Bower KJ, Clark RA, McGinley JL, Martin CL, Miller KJ. Feasibility and efficacy of the Nintendo Wii gaming system to improve balance performance post- stroke: protocol of a phase II randomized controlled trial in an inpatient rehabilitation setting. Games Health J 2013;2:103-8.

20. Choi D, Choi W, Lee S. Influence of Nintendo Wii Fit balance game on visual perception, postural balance, and walking in stroke survivors: a pilot randomized clinical trial. Games Health J 2018;7:377-84.

21. Worms G, Gollee ZM, Cikajlo I, Goljar N, Hunt KJ. Sensory electrical nerve stimulation for training dynamic balance responses in a chronic stroke patient. J Med Biol Eng 2011;31:19-29.

22. Altman DG. Missing outcomes in randomized trials: addressing the dilemma. Open Med 2009;3:e51-3.

23. Cattaneo D, Jonsdottir J. Sensory impairments in quiet standing in subjects with multiple sclerosis. Mult Scler 2009;15:59-67.

24. Saadat M, Salehi R, Negahban H, Shaterzadeh MJ, Mehravar M, Hessam M. Postural stability in patients with non-specific chronic neck pain: a comparative study with healthy people. Med J Islam Repub Iran 2018;32:33.

25. Lemay JF, Nadeau S. Standing balance assessment in ASIA D paraplegic and tetraplegic participants: concurrent validity of the Berg Balance Scale. Spinal Cord 2010;48:245-50.

26. Wirz M, Müller R, Bastiaenen C. Falls in persons with spinal cord injury: validity and reliability of the Berg Balance Scale. Neurorehabil Neural Repair 2010;24:70-7.

27. Ditunno JF Jr, Ditunno PL, Scivoletto G, et al. The Walking Index for Spinal Cord Injury (WISCI/ WISCI II): nature, metric properties, use and misuse. Spinal Cord 2013;51:346-55.

28. Jang Y, Hsieh CL, Wang YH, Wu YH. A validity study of the WHOQOL-BREF assessment in persons with traumatic spinal cord injury. Arch Phys Med Rehabil 2004;85:1890-5.

29. Saxena S, Chandiramani K, Bhargava R. WHOQOLHindi: a questionnaire for assessing quality of life in health care settings in India. World Health Organization Quality of Life. Natl Med J India 1998;11:160-5.

30. Elion O, Sela I, Bahat Y, Siev-Ner I, Weiss PL, Karni A. Balance maintenance as an acquired motor skill: delayed gains and robust retention after a single session of training in a virtual environment. Brain Res 2015;1609:54-62. 